\title{
ACCURACY EVALUATION OF STEREO CAMERA SYSTEMS WITH GENERIC CAMERA MODELS
}

\author{
Dominik Rueß, Andreas Luber, Kristian Manthey and Ralf Reulke \\ German Aerospace Center \\ Rutherfordstraße 2 \\ 12489 Berlin-Adlershof, Germany \\ (Dominik.Ruess,Andreas.Luber,Kristian.Manthey,Ralf.Reulke)@dlr.de
}

\author{
Commission V/5
}

KEY WORDS: Reconstruction, Geometry, Accuracy, Generic Cameras, Camera Model, Wide Baseline

\begin{abstract}
:
In the last decades the consumer and industrial market for non-projective cameras has been growing notably. This has led to the development of camera description models other than the pinhole model and their employment in mostly homogeneous camera systems. Heterogeneous camera systems (for instance, combine Fisheye and Catadioptric cameras) can also be easily thought of for real applications. However, it has not been quite clear, how accurate stereo vision with these cameras and models can be. In this paper, different accuracy aspects are addressed by analytical inspection, numerical simulation as well as real image data evaluation. This analysis is generic, for any camera projection model, although only polynomial and rational projection models are used for distortion free, Catadioptric and Fisheye lenses. Note that this is different to polynomial and rational radial distortion models which have been addressed extensively in literature.

For single camera analysis it turns out that point features towards the image sensor borders are significantly more accurate than in center regions of the sensor. For heterogeneous two camera systems it turns out, that reconstruction accuracy decreases significantly towards image borders as different projective distortions occur.
\end{abstract}

\section{INTRODUCTION}

Classical projective cameras have long been subject to stereo vision. Camera self-calibration, automated relative orientation, stereo reconstruction and many more issues have been very successfully worked on. With the introduction of panoramic or wide angle cameras several models have been developed, which are able to cope with the non-projective nature of many of these types of cameras, some of which are several trigonometric models for Fisheye lenses and catadioptric projection models. To prevent having to use different camera models within one application of heterogeneous cameras, generic camera models like the polynomial model, division model, rational model and shifted sphere model have been introduced. Furthermore, some approaches to perform stereo reconstruction on different types of cameras have been suggested, resulting in particular Epipolar models yielding curves instead of Epipolar lines. The results of these contributions quite often lack comprehensive investigations into reconstruction accuracy, as it is often required in photogrammetry. This paper investigates the whole process of $3 \mathrm{D}$ reconstruction with the above mentioned generic camera models. The main focus here is the influence of the radial distance to the projection center.

The next section will provide a short overview of existing approaches and camera models. This will be followed by a short review on stereo computation used for this paper. Afterwards, the results of analytical, numerical and real image data tests will be presented and evaluated.

Throughout this paper two camera systems will be used. As for the naming convention, lower case letters $\vec{x}$ will describe image points, upper case letters $\vec{X}$ are used for three dimensional object points and left-right image differentiation will be done with or without a hyphen $\overrightarrow{x^{\prime}}$ for the left and right image, respectively. The subscript $d$ will describe an entity within the distorted domain. If not stated differently, units are measured in millimeters.

\section{CAMERA MODELS OVERVIEW}

There are different models for describing the imaging geometry of a two camera system.

In Photogrammetry the imaging process can be modeled by means of the collinearity equations, see (Kraus, 2004). A world point $\vec{X}$ is mapped to an image point by subtracting the camera center $\vec{C}$ first, followed by rotating with the orientation matrix $R$. This includes $c$, the focal length and $x_{0}, y_{0}$ are the camera center offset. The resulting image vector $\vec{x}$ describes to location on the sensor.

\subsection{Radial Distortion}

This model describes the imaging process of distortion free cameras very accurately. It is also known as the pinhole model. Unfortunately, most of the cameras come with significant distortion towards the border regions of the image sensor. The effect of a camera lens usually results in pincushion or barrel distortion.

To overcome the accuracy issues introduced with different types of distortion, different models have been developed. In Photogrammetry, the Brown model is a very famous one (Brown, 1971). Most importantly it handles affinity, shear and tangential and radial distortion. The radial distortion is modeled by a polynomial, which maps the incoming radius to a radius on the sensor, which corresponds to the pinhole radius of the incoming ray.

Generally, a radial distortion function $L\left(r_{d}\right)$ converts a measured, real radius to a correct pinhole model radius $r$. Both mapping directions exist in literature, distorted radius to undistorted and vice versa. In both cases inversion of the function usually is not a trivial task to perform.

Many camera models have been developed in the last decades. Most of the models focus on improving the radial distortion aspect of the imaging process. It has turned out, that the division models tend to have an good approximation ability (Fitzgibbon, 
2001). The follow-up were rational models (Ma et al., 2004) where the function is a division of two polynomials. Lately, (Ricolfe-Viala and Sanchez-Salmeron, 2010) have discussed and analyzed the accuracy for modeling radius to radius mappings, including any type of division, polynomial and rational model. Refer to this paper for a comprehensive overview and description of radial distortion models.

\subsection{Varying the Projection Model}

All of the above methods have one major disadvantage; they cannot cope with wide-angle cameras with more than $180^{\circ}$ viewing angle. In these cases, different camera projections have been used. The work of Luber (Luber and Reulke, 2010) lists different possible projection models.

The idea of these approaches is to model the mapping of the inclination angle $\theta$ (between the connection of object point to camera center and the optical axis), to the resulting radius:

$$
r=f(\theta)
$$

If, without loss of generality, for a non-rotated and zero translated camera, an object point $\vec{X}$ is mapped to $\vec{x}$, the projection is:

$$
\begin{aligned}
& \vec{x}=\left\{\begin{array}{cl}
(0,0)^{\top} & , \text { if }\|\overrightarrow{\tilde{x}}\|<\epsilon \\
\overrightarrow{\vec{x}} f(\theta) & , \text { otherwise } \\
\|\overrightarrow{\vec{x}}\|
\end{array}\right. \\
& \text { where } \overrightarrow{\tilde{x}}=\left(\begin{array}{c}
X_{0} \\
X_{1}
\end{array}\right) \text {, from } \vec{X}=\left(\begin{array}{c}
X_{0} \\
X_{1} \\
X_{2}
\end{array}\right)
\end{aligned}
$$

One generic projection model has been introduced by Luber and Reulke (Luber and Reulke, 2010), namely polynomial mapping of inclination angle $\theta$ to camera chip radius $r$. Generally any of the above generic radius $r$ to radius $r_{d}$ distortion models can also be utilized as generic inclination angle $\theta$ to radius $r$ projection models. In (Luber and Reulke, 2010) and (Luber et al., 2012), different of these models are used as projection models and evaluated. Also, a method of calibrating such models is presented.

Note, if generic projection models are introduced, the radial distortion component can be discarded, as the projection implicitly involves the seeming distortion in the resulting images, see (Luber and Reulke, 2010) for more details.

\subsection{Stereo Accuracy Evaluation with Generic Projection Mod- els}

Camera calibration data of four types of lenses will be evaluated, including Fisheyes, Catadioptric cameras, weak and strong distortion regular lenses. With the use of generic camera models, $3 \mathrm{D}$ reconstruction for heterogeneous camera systems is possible by using one single model and varying parameters for each camera.

In general the inverse of a projection model has to be determined numerically, as exact solutions are either expensive or analytically not possible. Also note that the calibration of the cameras for this paper has been done in the fashion of (Luber et al., 2012), refer to this paper for the details.

\section{STEREO COMPUTATION OVERVIEW}

Multi-camera systems can almost always be broken down to a set of two different camera systems. For this reason we will stick to the two camera case throughout this paper.

In Photogrammetry, 3D reconstruction is known as space resectioning. The easiest case is to have a stereo normal situation where two cameras look towards the same direction with an alignment such that object points are imaged to the same $y$ coordinates in both cameras.

In the case of generic cameras it may not be useful to assume the normal case, as different types cameras may typically be positioned and aligned differently. Rectification may not be useful either, as it discards many of the image border areas.

The more general case of reconstruction means to intersect two skew lines or rays in three-dimensional space. In Kraus (Kraus, 2004), the general reconstruction case is based on a design matrix obtained from the collinearity equations. Generally the reconstructed point is the point of least distance to both rays. From our generic models, we directly obtain a base and a direction of the ray to the object. The base is the camera center $\vec{C}$ and the ray direction $\vec{d}$ is obtained from the inverse projection model, $\theta=f^{-1}(r)$ and the angle of the object point on the camera chip. Hence the ray $\tau_{\vec{x}}$ is described with:

$$
\begin{aligned}
& \tau_{\vec{x}}(\lambda)= \vec{C}+\lambda R^{-1} \vec{d}, \\
& \text { where } \vec{d}=\left\{\begin{array}{c}
\left(\begin{array}{c}
0 \\
0 \\
-1
\end{array}\right) \\
\left(\begin{array}{l}
x \cdot \frac{\sin (\theta)}{r^{r}(\theta)} \\
y \cdot \frac{\sin r}{r} \\
-\cos (\theta)
\end{array}\right),
\end{array},\right. \text {, otherwise } \\
& \text { and } r=\sqrt{x^{2}+y^{2} .}
\end{aligned}
$$

The object point $\vec{X}$, can be found with:

$$
\begin{aligned}
\vec{X} & =\frac{1}{2}\left(\tau_{\vec{x}}(\lambda)+\tau_{\vec{x}}^{\prime}\left(\lambda^{\prime}\right)\right), \\
\text { with }\left(\lambda, \lambda^{\prime}\right) & =\underset{\lambda, \lambda^{\prime}}{\operatorname{argmin}}\left(\left\|\tau_{\vec{x}}(\lambda)-\tau_{\vec{x}}^{\prime}\left(\lambda^{\prime}\right)\right\|\right) .
\end{aligned}
$$

Notice the $\epsilon$ in equation 3, which is a threshold below which the ray is supposed to be cast straight forward, from camera center to the distortion center on the image plane. Mathematically, this can be set to $\epsilon=0$. However, the incident angle to radius projection model involves a removable discontinuity around the image point $(0,0)^{\top}$, where we set the ray to $(0,0,1)^{\top}$. Unfortunately, it may behave numerically unstable around $(0,0)$. We will test for numerical inaccuracies in the simulation part of the results section, also to determine a suitable $\epsilon$.

\section{ANALYSIS AND RESULTS}

This section splits into three parts. First of all, some analytic accuracy evaluations will be presented. These predictions will then be compared to and inspected with some simulated data. Lastly, there will be the result of some real texture based images.

\subsection{Cameras Parameters}

In this section examples, simulations and experiments are conducted with the following cameras: 1.: Distortion free camera, $1280 \times 1024$ pixels resolution, $0.005 \mathrm{~mm}$ pixel size and a focal length of $2.5 \mathrm{~mm}$ - This camera is an artificial one, for comparability; 2.: Catadioptric Camera, 1392x1040 pixels resolution, $0.0063 \mathrm{~mm}$ pixel size and $1.63 \mathrm{~mm}$ focal length; 3.: Fisheye Camera, 1280x960 pixels resolution, $0.00645 \mathrm{~mm}$ pixel size and $3.3 \mathrm{~mm}$ focal length; 4 .: Wide-angle camera, $640 \times 480$ pixels resolution, $0.0067 \mathrm{~mm}$ pixel size and a focal length of $2.62 \mathrm{~mm}$.

For all results of this section, the remaining internal parameters are discarded. From our experience, these parameters are not significant for reconstruction accuracy; the main effect on accuracy 
is due to radial distortion or projection, respectively. Also we assume calibration to be sufficiently accurate, such that this does not evoke additional significant reconstruction inaccuracies.

We have chosen to evaluate the rational and polynomial projection model, whichever fits better to the data. A fixed number of parameters for both projection models are used, here this is 5 . On the one hand, all cameras can be calibrated similarly accurate with 5 parameters and on the other hand, due to the simulation within all experiments, there is no deviation from the actual (simulated) projection.

\subsection{Accuracy Analysis}

The accuracy of a reconstructed 3D point is influenced by different quantities. For this paper the following reasons have been identified: Baseline: Wider baselines allow for more accurate reconstructions; Scene distance: Scenes with a larger camera distance suffer from reconstruction inaccuracies. This correlates with the baseline; Measurement noise: Image points, determined automatically or manually, differ from correct projections; Resolution and pixel size: The higher the resolution the more accurate image points can be identified; Tangential distortion error: radially tangential localization errors will decrease the reconstruction errors towards boundary regions of the image sensor (see below); Radial distortion: If an optical system is subject to radial distortion or non-pinhole projection, basic geometric shapes will not transform to the same type of shape on the sensor; Calibration accuracy: Uncertainties in camera calibration will lead to inaccuracies in reconstruction.

From Computer Vision comes the notion of Epipolar lines, which handles the arbitrarily oriented camera case (Hartley and Zisserman, 2004), containing the stereo normal case as a special case. It is mostly used as a base for thresholds, i.e. for matching of features, where a distance of at most $x$ pixels from the Epipolar line implies a possible match.

In this paper another method is utilized: probability distributions of reprojected reconstructed 3D points. For this to be successful, there is the need for a proper investigation of the radial projection components. The projection function $f$ will not be linear, in most cases. But the fact that optical lenses have a very smooth surface makes it easy to locally assume linearity. Assume a uniform

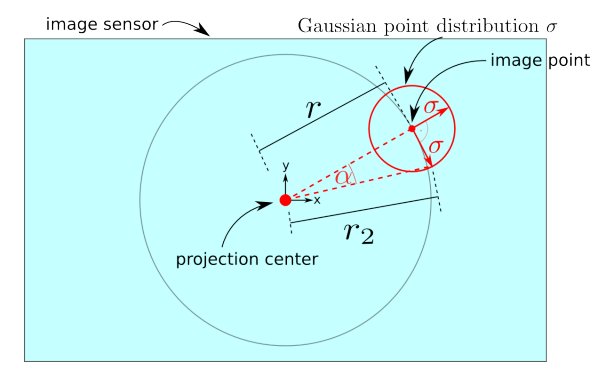

Figure 1: Image distribution; Illustrated Gaussian image distribution (exaggerated). As it is circular, the axes can be chosen arbitrarily, here perpendicular and tangential to the circle. Note that for the radial axis, only the radius and hence, the angle $\theta$ changes. For the tangential axis, the radius $r$ increases to $r_{2}$ (again: also $\theta$ ) as well as the angle $\alpha$ on the sensor.

normal 2D distribution $\sigma$ of an interest point selection around the correct image point. In this paper $\sigma$ will always be measured in pixel units, as this is the limiting factor for accuracy. This is a reasonable assumption as in the image domain, the ability to localize a point feature only depends on the pixel sizes.

The following methods work well for equal pixel sizes as the covariance matrix of this distribution describes a circle. This is for automated detection as well as for manual selection of interest points, which both usually are sub-pixel accurate. In figure 1 you can recognize two different axes of the distribution. At the respective interest points, one is perpendicular to the circle around the center of projection and the second one is tangential. As the uniform distribution is a circle, one may choose the principal axis arbitrarily (but still orthogonal).

Given the local assumption of linearity of $f$ and the selected perpendicular axis of the uniform distribution, at a given interest point $\vec{p}$, with projection radius $r$, we obtain:

$$
\sigma_{\theta}(r)=\frac{1}{2}(f(r+\sigma)-f(r-\sigma)) .
$$

For small $\sigma$, this converges to the derivation of $f$ :

$$
\sigma_{\theta}(r)=\sigma \frac{d f(r)}{d r}=\sigma f^{\prime}(r)
$$

hence the mapping of the perpendicular axis of the Gaussian can be approximated with equation 6 .

The mapping of the second axis $\sigma \mapsto \sigma_{t}$, tangential to the circle with radius $r$, can also be assumed linear for small $\sigma$. It is slightly more difficult, as it involves increasing angles in the image plane but also increasing angles due to locally increasing the radius. Let $\overrightarrow{x_{r}}$ be the point $\vec{x}$ translated radially, by $\sigma$ times the pixel size. Let further $\overrightarrow{x_{t}}$ be the point $\vec{x}$ translated tangentially, by the same amount.

$$
\sigma_{t}=\arccos \left(\left(\tau_{\overrightarrow{x_{r}}}(1)-\vec{C}\right) \cdot\left(\tau_{\overrightarrow{x_{t}}}(1)-\vec{C}\right)\right)
$$

For many cameras, the first axis with standard deviation $\sigma_{\theta}$, will produce larger errors to boundary regions, as the angular difference increases for most cameras, towards the imaging sensor boundaries.

The second axis, $\sigma_{t}$ will decrease with increasing radius. This is because the angular difference of the inverse $f^{-1}(\theta)$ decreases with increasing radius.

To illustrate the different effects, we created a simulation setup where the cameras are rotated such that a fixed object point creates a trace on the image plane, see figure 2 . In figure 3 these two different effects are plotted for $\sigma=0.1$. Note, how the radial error (solid lines) increases for the Fisheye and the wide-angle lenses but decreases for the Catadioptric and the distortion free cameras.

The tangential error decreases with increasing radius, for all of the optical systems, roughly compensating the effect of the radial error for Fisheye and wide-angle lenses. Which of the two ef-

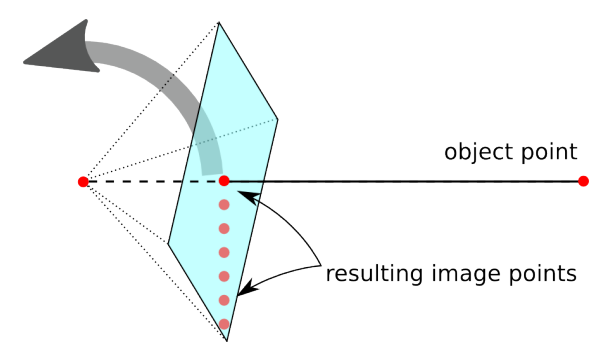

Figure 2: Illustration of Rotation; The rotation is applied at equidistant angle steps, such that a trace is created on the image plane, diagonal within the sensor rectangle.

fects has a greater impact depends on the camera parameters, but for our data, the overall error will usually decrease towards the boundary regions of the image sensors.

To make sure, our assumption of local linearity is a good one, we illustrate the predicted and measured mapping of the Gaussian 


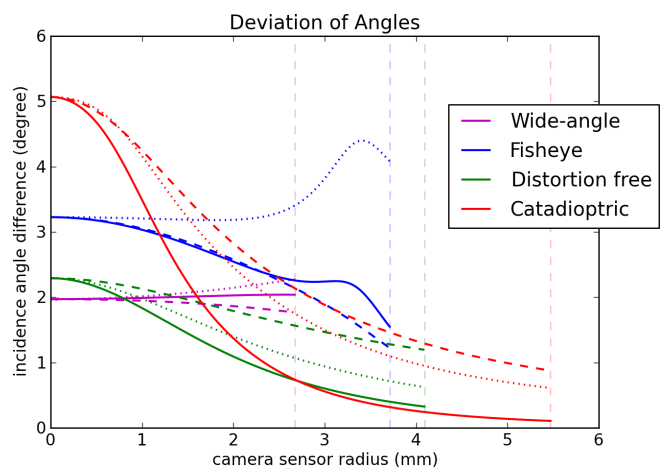

Figure 3: Influence of Radial and Tangential Error Distribution; The graph shows the influence of the two axes identified in the text: radial error distribution (dotted lines) and tangential error distribution (dashed lines). The solid line illustrates the overall effect, by computing the volume for the respective ellipse. $\sigma=0.1$ in pixel units. Vertical dashed lines denote maximal possible sensor radius.

point error to an ellipse at $1000 \mathrm{~mm}$ distance. This can be found in figure 4 . The Gaussian distribution with axes $\sigma_{\theta}$ and $\sigma_{t}$ can be
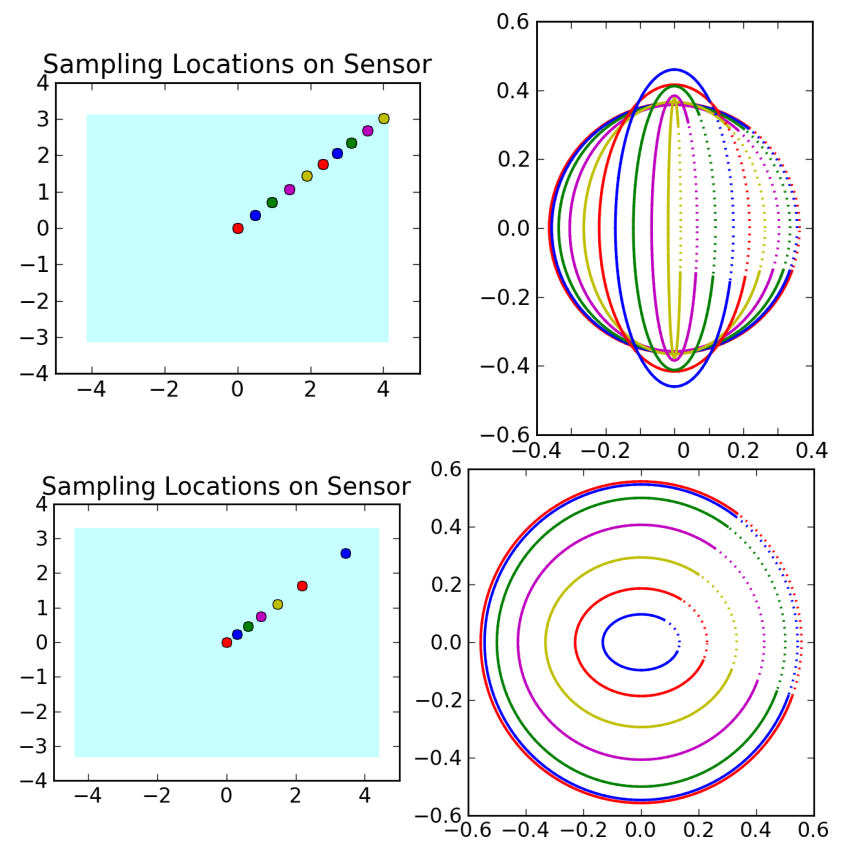

Figure 4: Predicted and Measured Error Ellipses; The top graph shows the Fisheye lens, below is the Catadioptric camera. Error Ellipses at $1000 \mathrm{~mm}$ distance, predicted (dashed lines) by determining the perpendicular and tangential axis distances. Measured (solid lines) by sampling in the image plane with $\sigma=0.1$ pixel units, followed by a projection to space. The location of these samplings is illustrated in the blue area, which represents the camera sensor. Location circles on sensor are exaggerated for sake of visibility. All units in $\mathrm{mm}$.

cast to 3D space, resulting in a cone shape with its apex at camera center $\vec{C}$ and its base the elliptical standard deviation.

\subsection{Simulation With Real Camera Parameters}

In this part of the result section some simulation results are presented. These are mainly results of reconstruction with simulated noise within the image interest point locations.
4.3.1 Center Point Discontinuity As mentioned above, imaging of points may result in numerical instability, around image point $(0,0)^{\top}$. This can be seen for the projection and the reconstruction case, see equation 2 and 3 , respectively. The simulation has shown that this concern is not confirmed, as all image points converge to zero, for input radii down to $10^{-18}$. To $(0,0)^{\top}$, exactly. On the other hand, the inversion needs to be investigated.

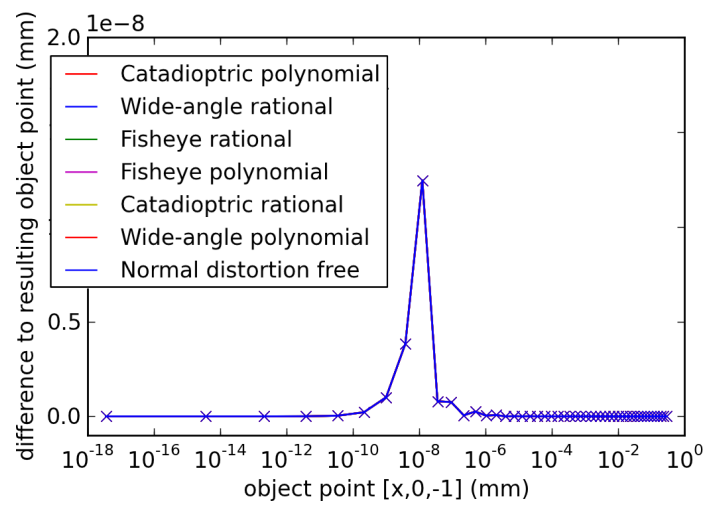

Figure 5: Numerical Stability Test; When back-projected, the lower graph shows the distance to the original points around zero.

Mapping radius to a direction vector/ray involves numerically inverting the projection model. In equation 3 a very similar $\epsilon$ occurs. As you can see in figure 5, with our current implementation of the inverse computation of $f$ (the Python lib SciPy: fsolve), there are some minor instabilities. However, these are very small, neglectable.

These experiences may lead to set the thresholds to $\epsilon=10^{-10}$, for instance, to avoid division by zero in cases where object points image exactly to $(0,0)^{\top}$ and vice versa.

4.3.2 Overall Error and Effect of different baselines Just like the illustration in figure 2, we rotate all the cameras such that the diagonal elements are imaged. We sample a given $\sigma$ Gaussian distribution around the currently project image point. This image point was projected from a fixed object point $\vec{X}=$ $(0,0,-1000)^{\top}$. The second camera is an optimal, error free camera, moved along the $X$-axis with a given baseline. This allows for evaluation of the first camera, only. Figure 6 shows, that indeed, the smallest baseline produces the largest error, though this is not a surprise. More importantly, errors decrease with image points towards the image sensor boundaries. This effect is a very dramatic one for the Catadioptric and the distortion free camera. Overall, this result confirms the earlier analytical prediction, where tangential and perpendicular errors roughly add up to the final error.

\subsection{Real Image Data}

The above analyses consider fixed $\sigma$ positional noise, only. This gives a very nice theoretical illustration of the problem. Without changing any of the considerations, the points may have different individual $\sigma$. This theory may be applied for (manual) point detectors, for instance.

However, automated interest point detectors are actually slightly more region based, due to scale-space detection and neighborhood sampling. Probably, this results in a radius dependent positioning accuracy.

4.4.1 Automated Feature Detection and Matching Usually, feature matching is done based on the feature descriptor distance and the constraints of Epipolar geometry. This involves applying the fundamental matrix $F$, see (Hartley and Zisserman, 2004), 


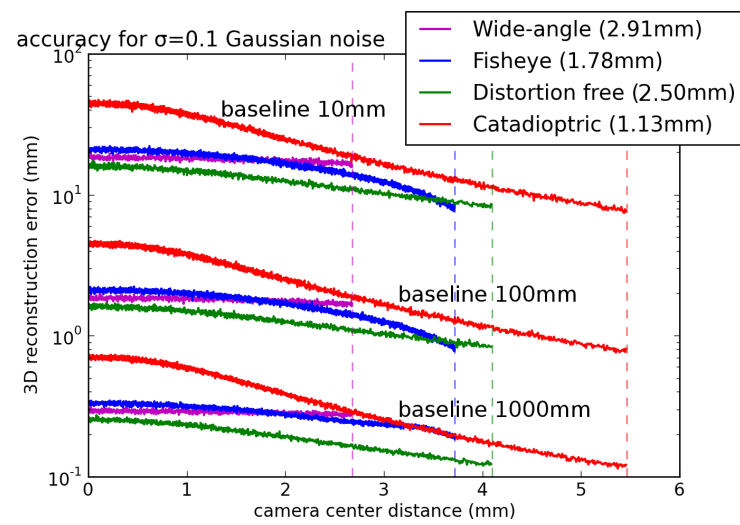

Figure 6: Different Baselines; This figure illustrates the effect of different baselines on 3D reconstruction. The graph shows the simulation for a $\sigma=0.1$ pixel units. Plotted are the average reconstruction error values for each radius.

for instance. The Epipolar geometry reduces the search domain from a two dimensional space to a one-dimensional one.

However, here this involves numerically inverting the projection and other difficulties such as finding the closest point on the projection curve. Hence, one might as well use a different approach, which implicitly models the Epipolar constraint.

In the following, the approach is illustrated: First of all, potential matches are obtained, utilizing an approximately nearest neighbor search (ANN), based on feature distances. For all these potential matches compute the reconstruction in space and reproject to both cameras. Similar to the distance of both rays to the reconstructed point, a score can be evaluated in the image based on the given distribution $\sigma$.

Obviously, if both image points are subject to the Epipolar geometry, the reprojection will map to the original points, exactly. If the Epipolar constraint is violated, the reprojection will move away from the original point. Given the original image points $\vec{x}$, $\overrightarrow{x^{\prime}}$, the reconstructed point $\vec{X}$ and the reprojected image points $\overrightarrow{x_{p}}, \overrightarrow{x_{p}^{\prime}}$, a score can be defined as:

$$
s(\vec{X})=G_{\vec{x}, \sigma}\left(\overrightarrow{x_{p}}\right) \cdot G_{\overrightarrow{x^{\prime}, \sigma^{\prime}}}\left(\overrightarrow{x_{p}^{\prime}}\right)
$$

where $G_{\vec{x}, \sigma}$ is the Gaussian density with $\sigma$ around $\vec{X}$. The normalized version of the score, here

$$
s_{n}(\vec{X})=\frac{s_{n}(\vec{X})}{G_{\vec{x}, \sigma}(\vec{x}) \cdot G_{\overrightarrow{x^{\prime}}, \sigma^{\prime}}\left(\overrightarrow{x^{\prime}}\right)},
$$

can be used, together with a threshold $t$ to decide, whether a potential match fulfills Epipolar geometry. We have determined $\sigma=0.5$ and $t=0.01$ to obtain matches with separation of just more than 1 pixel from Epipolar geometry.

A SURF feature descriptor was used in combination with a Harris corner detector, see (Mikolajczyk and Schmid, 2004) for a thorough overview of interest point detectors. To obtain a high number of interest point, a low threshold was used for the detection (i.e. Hessian was set to 50).

4.4.2 Evaluation Evaluating interest point detectors mainly involves evaluating the completely automated feature detection, matching and reconstruction process. Obviously there is a need for accurate ground truth data. There are different possible approaches. On approach is a real scene, with depth is measured with a second sensor, i.e. a laser. A different approach is to use well known three dimensional shapes. We chose for a different approach: Simulation. The cameras are placed in the center of a cube with side length of $2000 \mathrm{~mm}$. Different textures (wood, forest, urban, etc...) are used for different simulation iterations. The resections of automatically detected features can now be evaluated with perfectly known ground truth. This approach is illustrated in figure 7.
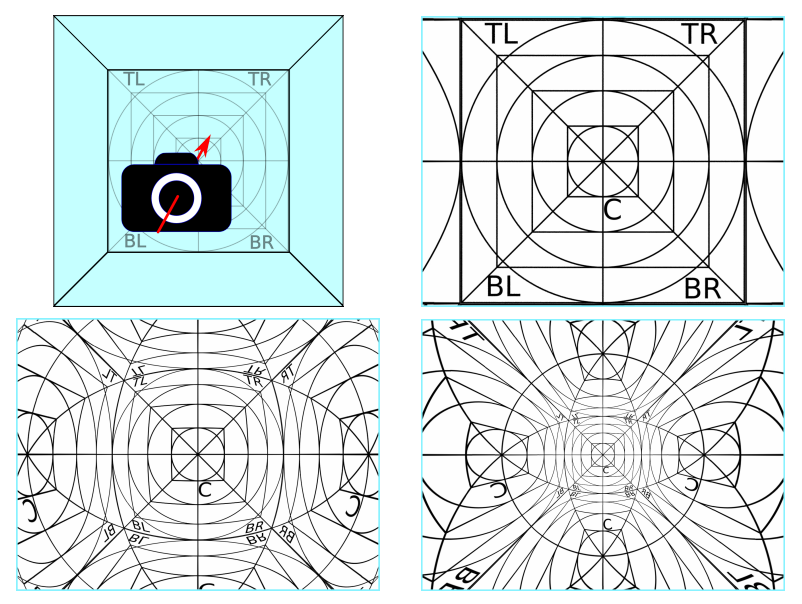

Figure 7: Illustration of Simulation; The top left image illustrates the camera placement. The top right image is the undistorted camera image. The lower row contains a Fisheye image (left) and a Catadioptric image (right)

For each camera, different positions were sampled at: $(0,0,0)^{\top}$, the position to be compared to; $(10,0,0)^{\top}$, a small baseline position, looking to negative $Z$ axis; $(100,0,0)^{\top}$, a larger baseline position looking to negative $Z$ axis; $(500,500,-500)^{\top}$, a wide baseline position, looking to the center of the front cube face, $(0,0,-1000)^{\top}$.

Now different properties of the system can be evaluated: the quality of matching (i.e. number of correct matches), the accuracy of reconstructed interest/feature points, the influence of the baseline length, the influence of perspective distortion and possibly influence of radial distance to projection center. In table 1 and 2 some

Table 1: Reconstruction Statistics for Different Camera Systems: The table lists the results of reconstruction. Errors of $>100 \mathrm{~mm}$ were considered false matches. Displayed are median errors $(\mathrm{mm})$, which gives a good indication of the overall performance. Numbers are accumulated over different textures.

\begin{tabular}{c||c|c|c} 
Baseline: & Small & Larger & Wide \\
Constellation & $(10,0,0)$ & $(100,0,0)$ & $10^{2} \cdot(5,5,-5)$ \\
\hline \hline Normal/Normal & 0 & 0 & 3.7 \\
Normal/Catad. & 46.3 & 28.7 & 4.1 \\
Normal/Fisheye & 49.1 & 16.8 & 3.1 \\
Catad./Catad. & 27.7 & 14 & 9.4 \\
Catad./Fisheye & 49.8 & 32.3 & 9.3 \\
Fisheye/Fisheye & 29.7 & 11.8 & 6.6
\end{tabular}

of the answers are given. For Fisheye and Catadioptric cameras a small baseline is too small, most likely due to the compressed projection of object points at the image center. Fisheye and Catadioptric cameras perform similarly well. They have the least error in wide baseline situations. However, the number of matches as well as the ratio of good/bad matches is best in the medium baseline situation. This is likely due to a larger overlapping field of view and similar distortion of corresponding features in these situations.

This same argument holds for the Normal/Normal case, were the 
Table 2: Number of Matches for Different Camera Systems: This table lists the number of correct (error $<100 \mathrm{~mm}$ ) matches vs the number of wrong matches.

\begin{tabular}{c||c|c|c} 
Const/Base & Small & Larger & Wide \\
\hline \hline Norm/Norm & $79677 / 6321$ & $84468 / 346$ & $6004 / 4254$ \\
Norm/Catad & $364 / 3103$ & $2585 / 1211$ & $5866 / 1440$ \\
Norm/Fish & $3998 / 11387$ & $12241 / 4078$ & $8644 / 3131$ \\
Catad/Catad & $56712 / 27514$ & $61263 / 6147$ & $5223 / 2634$ \\
Catad/Fish & $1937 / 12889$ & $10981 / 6710$ & $7653 / 2858$ \\
Fish/Fish & $50911 / 24410$ & $59520 / 5775$ & $10148 / 4053$
\end{tabular}

error increases unexpectedly for wide baseline situations. To answer the question of radial distortion and perspective influence on automatically detected features, we decided to evaluate the three homogeneous and heterogeneous camera systems. This means, at baseline distance of only $10 \mathrm{~mm}$ two cameras of the same type will likely not cause projective problems and follow the above mentioned theory for point features, whilst in the case of heterogeneous camera systems, the effect of different perspective distortion will arise and cause larger errors towards larger radii.

These suppositions are roughly confirmed by the results presented in figure 8. Especially for heterogeneous systems, the error in creases towards the sensor border, mainly due to different projective distortions.

For homogeneous systems, one can see the predicted decreasing effect for Catadioptric cameras. For both other systems, it is difficult to recognize a specific pattern.
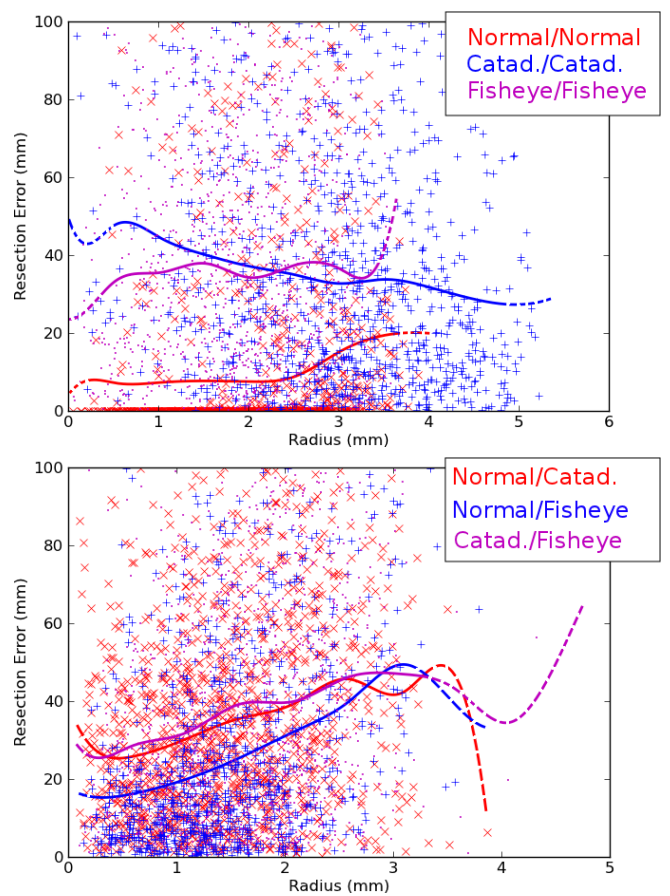

Figure 8: Error Evaluation of Two Camera Systems; The upper graph shows the error plot for homogeneous systems, which reflects similar projective distortion at roughly the same radial sensor distance (small base line). Below are heterogeneous systems, the maximum of both radii is used for plotting. Solid lines represent smoothed least squares fit of the data (Splines). Dashed parts are with little data. Not all of the data is plotted due to heavy cluttering of the graph.

\section{CONCLUSIONS AND FUTURE WORK}

This article presents accuracy analysis for stereo processing with generic camera projection models, with a main aspect on radially induced errors.

It has been shown, that for point features errors tend to decrease with larger radius from the projection center, for all types of cameras. Additionally, for two camera systems, the main influence for detectors like SURF is the differently distorted appearance of corresponding features. For heterogeneous camera features this means increase in reconstruction error for larger sensor radii. Another point to mention is the general decrease in accuracy for omnidirectional two camera systems, mainly because much more of the scene is imaged to the same image resolution.

The simulation hasn't considered additional error sources like possible overlaps, lighting difference, point spread functions and other influences. Fixed and exact camera parameters have been assumed. But if the uncertainty parameters are known it is possible to adapt the above theory by means of error propagation. Lastly, it might be useful to additionally compare different interest point detectors/descriptors.

\section{ACKNOWLEDGEMENTS}

Dominik Rueß and Kristian Manthey would like to acknowledge financial support of the Helmholtz Research School on Security Technologies.

\section{REFERENCES}

Brown, D., 1971. Close range camera calibration. Photogrammetric Engineering 37, pp. 855 - 866.

Fitzgibbon, A., 2001. Simultaneous linear estimation of multiple view geometry and lens distortion. In: Computer Vision and Pattern Recognition, 2001. CVPR 2001. Proceedings of the 2001 IEEE Computer Society Conference on, Vol. 1, pp. 125 - 132.

Hartley, R. I. and Zisserman, A., 2004. Multiple View Geometry in Computer Vision. Second edn, Cambridge University Press.

Kraus, K., 2004. Geometrische Informationen aus Photographien und Laserscanneraufnahmen. de Gruyter, Berlin.

Luber, A. and Reulke, R., 2010. A unified calibration approach for generic cameras. In: The international archives of the Photogrammetry, remote sensing and spatial information sciences, pp. $399-404$.

Luber, A., Rueß, D., Manthey, K. and Reulke, R., 2012. Calibration and epipolar geometry of generic heterogenous camerasystems. In: ISPRS World Congress Melbourne, in press.

Ma, L., Chen, Y. and L. Moore, K., 2004. Rational radial distortion models of camera lenses with analytical solution for distortion correction. IJIA.

Mikolajczyk, K. and Schmid, C., 2004. Scale \& affine invariant interest point detectors. International Journal of Computer Vision 60(1), pp. $63-86$.

Ricolfe-Viala, C. and Sanchez-Salmeron, A.-J., 2010. Lens distortion models evaluation. Appl. Opt. 49(30), pp. $5914-5928$. 